il est possible que l'interférence agisse ici exclusivement au niveau transcriptionnel. Ce ne peut pas être le cas avec un virus relativement proche, tel que celui d'Orsay, dont le génome n'existe pas sous une forme ADN.

\section{En conclusion}

L'extinction d'un gène par l'ARNdb correspondant peut se transmettre au fil des générations chez des animaux, comme chez les plantes et les champignons. Chez C. elegans, elle semble utiliser l'amplification de petits ARN spécifiques et la répression transcriptionnelle à chaque génération. L'impact de ce processus de transmission sur l'adaptation phénotypique des organismes en milieu naturel reste cependant à démontrer. Il est possible que la cible principale de cette extinction multigénérationnelle soit les transposons insérés dans le génome. $\nabla$

Multigenerational transmission of RNA interference in the nematode Caenorhabditis elegans

\section{LIENS D'INTÉRÊT}

Les auteurs déclarent n'avoir aucun lien d'intérêt concernant les données publiées dans cet article.

\section{REMERCIEMENTS}

L'équipe est financée par le CNRS, l'ENS, I'Inserm, et pour la recherche sur les virus, la subvention de l'ANRII BSV3 01301. Nous remercions Valérie Robert (ENS Lyon) pour ses commentaires.

\section{RÉFÉRENCES}

1. Fire A, Xu S, Montgomery MK, et al. Potent and specific genetic interference by double-stranded RNA in Caenorhabditis elegans. Nature 1998 ; 391 : 806-11.

2. Fischer SEJ. Small RNA-mediated gene silencing pathways in C. elegans. Int J Biochem Cell Biol 2010 ; 42 : 1306-15.
3. Vastenhouw NL, Brunschwig K, Okihara KL, et al. Gene expression: long-term gene silencing by RNAi. Nature $2006 ; 442: 882$.

4. Grishok A, Tabara H, Mello CC. Genetic requirements for inheritance of RNAi in C. elegans. Science 2000 ; 287 : 2494-7.

5. Alcazar RM, Lin R, Fire AZ. Transmission dynamics of heritable silencing induced by double-stranded RNA in Caenorhabditis elegans. Genetics 2008 ; 180 : 1275-88.

6. Burton NO, Burkhart KB, Kennedy S. Nuclear RNAi maintains heritable gene silencing in Caenorhabditis elegans. Proc Natl Acad Sci USA $2011 ; 108$ : 19683-8.

7. Gu SG, Pak J, Guang S, et al. Amplification of siRNA in Caenorhabditis elegans generates a transgenerational sequence-targeted histone $\mathrm{H} 3$ lysine 9 methylation footprint. Nat Genet 2012 ; 44 : 157-64.

8. Félix M-A, Ashe A, Piffaretti J, et al. Natural and experimental infection of Caenorhabditis nematodes by novel viruses related to nodaviruses. PLoS Biol $2011 ; 9$ : el000586.

9. Lu R, Maduro M, Li F, et al. Animal virus replication and RNAi-mediated antiviral silencing in Caenorhabditis elegans. Nature 2005 ; 436 : 1040-3.

10. Rechavi 0 , Minevich G, Hobert 0. Transgenerational inheritance of an acquired small RNA-based antiviral response in C. elegans. Cell 2011 ; $147: 1248-56$.

\section{NOUVELLE}

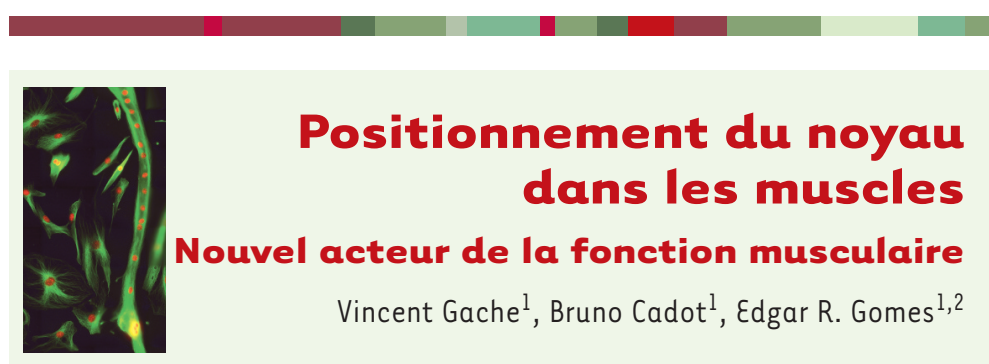

mant de manière coordonnée les protéines spécifiques du muscle. Les myoblastes deviennent alors des myocytes, cellules spécialisées ayant acquis la particularité de pouvoir fusionner entre elles et de conduire à la formation de myotubes contenant plusieurs noyaux. Ces myotubes sont caractérisés par un positionnement central des noyaux qu'ils contiennent. Au cours de la maturation des myotubes, des unités contractiles appelées sarcomères vont se former par l'association précise de réseaux d'actines et de moteurs moléculaires, les myosines, permettant la contraction des fibres musculaires. Ces myotubes matures deviennent des myofibres et localisent les noyaux à la
lUMR S 787 Inserm, université Paris 6 Pierre et Marie Curie, 75634 Paris, France ; ${ }^{2}$ Groupe hospitalier Pitié-Salpêtrière, institut de myologie, 105, boulevard de l'Hôpital, 75634 Paris Cedex 13, France. edgar.gomes@upmc.fr.

V. Gache et B. Cadot ont contribué équitablement à ce travail.

périphérie, près de la membrane. Ces myofibres sont les briques élémentaires du muscle [1, 2].

In vivo, tandis que dans les fibres matures les noyaux se localisent à la périphérie, ils sont alignés au centre de la fibre régénérée (Figure IA, B) [3]. Afin d'étudier ce phénomène, nous avons reproduit en laboratoire les premières étapes de la formation des fibres musculaires, et nous avons suivi les déplacements des noyaux au cours du temps. Nous avons pu observer qu'à la suite de la fusion d'un myocyte avec un myotube, le noyau du myocyte migre rapidement vers le centre du myotube. Par la suite, ces noyaux s'alignent le long du syncytium (Figure 1C). Ces observations nous ont

Photo : myotube formé in vitro après 5 jours de differen tion (microtubules en vert, noyaux en rouge) (c V. Gache, UMR S 787 Inserm) 


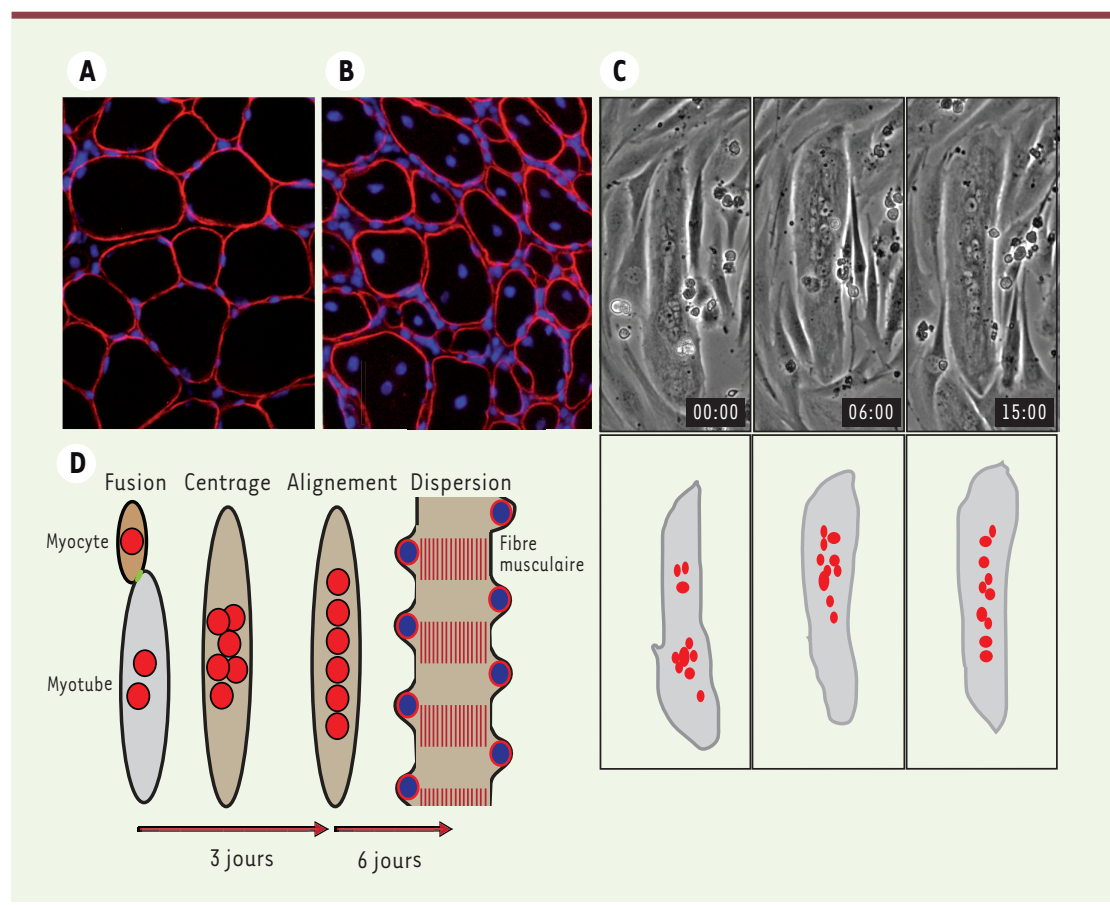

Figure 1. Positionnement des noyaux dans les fibres musculaires. A, B. Muscles et noyaux in vivo: exemple d'une coupe transversale d'un muscle sain ( $A$ ) ou d'un muscle en régénération ( $B$ ). Les noyaux apparaissent en bleu. Le contour des fibres apparaît en rouge grâce à un marquage par anticorps fluorescent dirigé contre la laminine. C. Alignement des noyaux dans des myotubes formés in vitro : photographies en contraste de phase tirées d'une vidéo focalisée sur un myotube (haut ; les nombres indiquent le temps en min) et schémas du positionnement des noyaux dans les myotubes (bas). D. Prédiction des différentes localisations des noyaux au cours de la maturation de la fibre musculaire.

conduits à identifier différentes étapes dans le positionnement des noyaux lors de la formation et la maturation des fibres musculaires (Figure 1D). La mauvaise localisation des noyaux dans les fibres est une caractéristique majeure de nombreuses maladies musculaires, et plus particulièrement des myopathies centronucléaires $[4,5]$.

\section{MAP7 et Kif5B sont responsables de l'alignement des noyaux dans les fibres musculaires}

Afin de déterminer les mécanismes responsables du positionnement des noyaux dans les muscles, nous avons criblé, chez des embryons de drosophiles, des mutations entraînant un changement de localisation des noyaux dans les fibres musculaires. Une mutation affectant le gène Ensconsin est responsable d'un changement de localisation des noyaux
(Kif5b ou Khc) [8]. Ces deux protéines étaient déjà suspectées d'interagir pour se lier aux microtubules dans un système in vitro [9]. L'absence de cette kinésine in vivo dans les muscles de larves de drosophiles, ou in vitro dans les myotubes formés à partir de cellules de souris, entraîne l'agrégation des noyaux de la même manière qu'en l'absence de MAP/ Ensconsin. L'interaction de ces deux protéines a été démontrée in vitro dans des expériences d'immunoprécipitation. Le rôle de cette interaction dans la localisation des noyaux dans les fibres a été confirmé par l'expression d'une protéine chimérique, qui mime l'interaction des deux protéines et est capable de réaligner les noyaux dans les fibres musculaires en l'absence de MAP7 dans les muscles formés in vitro [6]. Ces résultats ont permis d'élaborer un modèle dans lequel l'interaction de MAP7 avec Kif5b permet de stabiliser les microtubules émanant de la membrane des noyaux des fibres musculaires de façon antiparallèle [10], et de permettre ainsi l'alignement et l'espacement des noyaux dans les fibres (Figure 2).

\section{Implication physiologique} du positionnement des noyaux dans les muscles

L'impact du mauvais positionnement des noyaux dans les fibres musculaires sur leur efficacité a été analysé in vivo chez la larve de drosophile en testant sa motilité. Ces expériences ont montré une baisse significative de la motilité des larves exprimant une forme mutée de la protéine MAP7/Ensconsin par rapport aux larves contrôles. Dans ces larves, seule la localisation des noyaux dans les muscles est affectée, suggérant une importante corrélation entre positionnements aberrants des noyaux dans les muscles et réduction de l'efficacité de la fonction musculaire [6].

\section{Applications thérapeutiques}

Les patients atteints de maladies musculaires, telles que les myopathies centronucléaires, ont des difficultés 


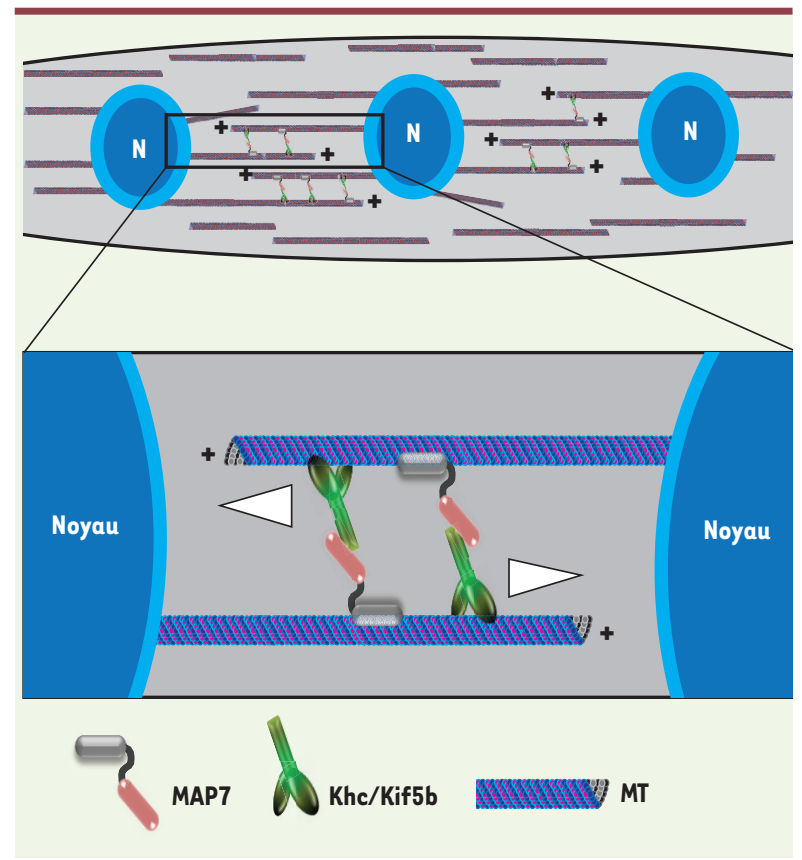

Figure 2. Modèle de l'alignement des noyaux. Dans un myotube contenant plusieurs noyaux (N), les microtubules émanant des différents noyaux forment des réseaux antiparallèles reliés entre eux par le complexe MAP7/ Kif5b. D'un côté, MAP7 se lie à un microtubule (MT) et Kif5b se lie à un autre microtubule de manière antiparallèle. Au contraire de MAP7, Kif5b a la possibilité de se déplacer vers l'extrémité des microtubules établissant ainsi un jeu de forces entre différentes paires de microtubules antiparallèles. Ce jeu de forces est impliqué dans l'espacement et l'alignement des noyaux dans les myotubes.

motrices. Dans ces pathologies, un mauvais positionnement des noyaux dans les fibres musculaires est observé. Il n'est pas encore clair si les défauts de localisation des noyaux dans les fibres sont une cause de la faiblesse musculaire ou le résultat d'un dysfonctionnement des fibres. Toutefois, nos résultats montrent une corrélation entre le bon positionnement des noyaux dans les fibres et la fonction des muscles. Nous suggérons qu'une correction des défauts de positionnement des noyaux musculaires chez des patients atteints de myopathies pourrait entraîner une amélioration de leur fonction musculaire. $\diamond$ Spatial distribution of nuclei in muscle fibers: a novel actor of muscular function

\section{LIENS D'INTÉRÊT}

Les auteurs déclarent n'avoir aucun lien d'intérêt concernant les données publiées dans cet article.

\section{RÉFÉRENCES}

1. Carlson BM. Muscle regeneration in amphibians and mammals: passing the torch. Dev Dyn 2003; 226 : 167-81.

2. Dhawan J, Rando TA. Stem cells in postnatal myogenesis: molecular mechanisms of satellite cell quiescence, activation and replenishment. Trends Cell Biol 2005 ; 15 : 666-73.

3. Bruusgaard JC, Liestø| K, Ekmark M, et al. Number and spatial distribution of nuclei in the muscle fibres of normal mice studied in vivo.J Physiol (Lond) 2003; 551 : 467-78.

4. Cohn RD, Campbell KP. Molecular basis of muscular dystrophies. Muscle Nerve 2000 ; 23 : 1456-71.

5. Jungbluth $\mathrm{H}$, Wallgren-Pettersson C, Laporte J. Centronuclear (myotubular) myopathy. Orphanet) Rare Dis $2008 ; 3: 26$.

6. Metzger T, Gache V, Xu M, et al. MAP and kinesin-dependent nuclear positioning is required for skeletal muscle function. Nature 2012 ; 484 : 120-4.

7. Masson $D$, Kreis TE. Identification and molecular characterization of $\varepsilon$-MAP-115, a novel microtubule-associated protein predominantly expressed in epithelial cells. J Cell Biol 1993; 123 : 357-71.

8. Vale RD, Reese TS, Sheetz MP. Identification of a novel force-generating protein, kinesin, involved in microtubule-based motility. Cell $1985 ; 42$ : $39-50$.

9. Sung HH, Telley IA, Papadaki P, et al. Drosophila ensconsin promotes productive recruitment of Kinesin-1 to microtubules. Dev Cell 2008; 15 : 866-76.

10. Tassin AM, Maro B, Bornens M. Fate of microtubuleorganizing centers during myogenesis in vitro. J Cell Biol $1985 ; 100: 35-46$.

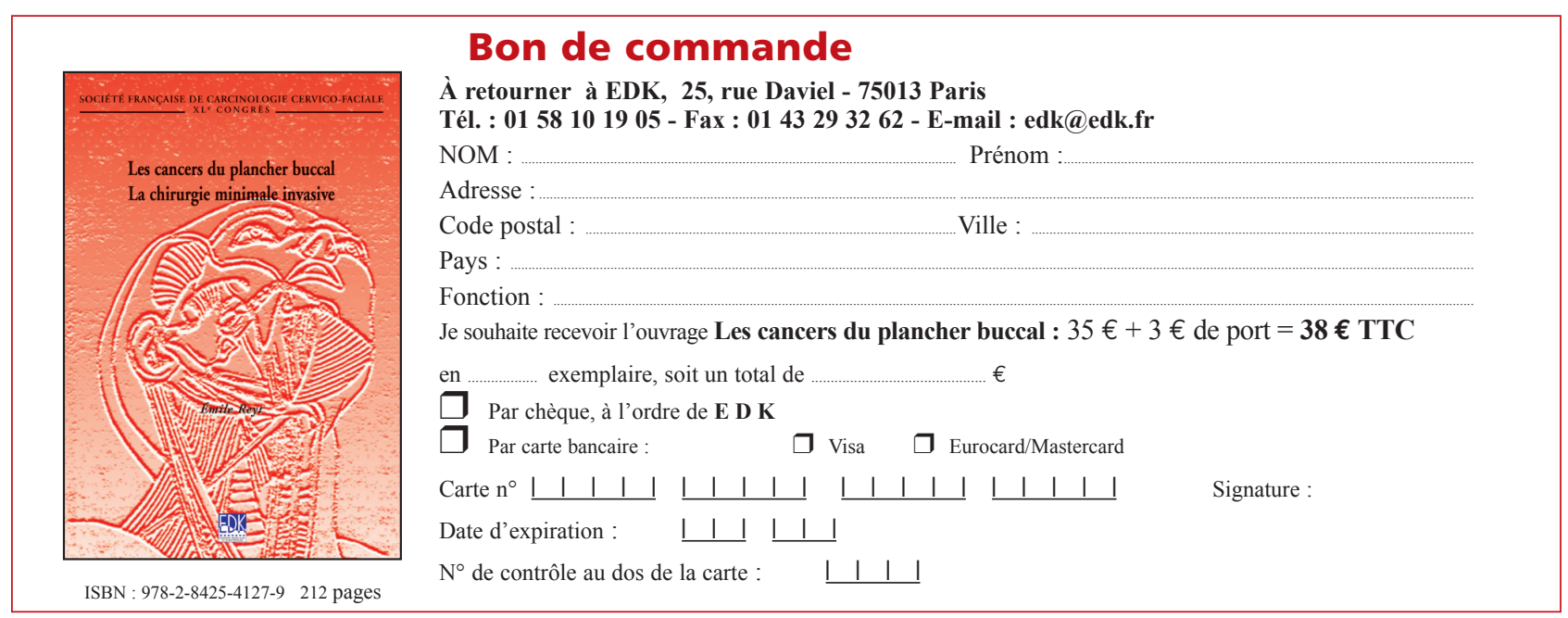

